\title{
A RECONFIGURABLE OPTOFLUIDIC DEVICE FOR ADAPTIVE IMAGING AND POSITION ESTIMATION WITH A WIDE FIELD OF VIEW
}

\author{
Hanyang Huang, and Yi Zhao* \\ The Ohio State University, Columbus, Ohio, USA
}

\begin{abstract}
Imaging with a wide field-of-view (FOV) is important for versatile applications such as surveillance, robotic vision, and laparoendoscopic imaging. Artificial compound eyes that imitate the insect compound eyes can provide a wide FOV and have a compact size. However, current artificial compound eye devices either require curved photodetectors, have substantial image distortion, or lack self-accommodation capability. In this paper, we present a reconfigurable optofluidic lens that incorporates the architectural merits of the Xenos peckii compound eye and the mammalian eye into one system. The device can provide adaptive imaging and position estimation capability with a wide FOV using a planar image sensor.
\end{abstract}

\section{INTRODUCTION}

Insect compound eye is a unique and sophisticated optical system that excels in its compact design, wide FOV and highly sensitive motion detection capability $[1,2]$. Artificial compound eye devices that imitate the wide-angle vision of natural counterparts have shown enormous advantages in various applications including robotic vision, photography, surveillance, medical imaging, motion detection, etc. [3-7]. Several types of artificial compound eye have been developed based on different configurations. One design imitates the compound eye of fruit fly Drosophila [8-10]. A solid microlens array is arranged on a hemispherical surface. Each microlens faces towards a different field angle. This design can offer a FOV as large as $160^{\circ} \times 180^{\circ}$. However, a curved image sensor is normally required to accommodate the curved image planes. The fabrication and alignment process are fairly complicated and challenging $[11,12]$. Another design imitates the compound eye of Xenos peckii [13-15]. Such device consists of a planar solid microlens array and a planar aperture array. The light beams focused by the microlenses pass through the aperture array and form the images on a planar image sensor. The fabrication and assembly complexities are greatly reduced. The FOV is, however, fairly limited due to the planar arrangement of microlenses. Several additional efforts have also been put in to combine the architectural merits of the above two designs [16, 17]. The microlenses are arranged on a hemispherical surface and an optical replay component is placed in front of the aperture array to steer the light beams on a planar image sensor. Although such design modestly improves the FOV, the maximally obtained FOV is still not sufficient for wide-angle imaging.

These artificial compound eyes also suffer the poor depth perception capabilities, poor image quality and low signal-to-noise ratio (SNR) of their natural counterparts, due to that each small lens only has a small lens aperture [18]. Mammalian eye, another vision system in nature, offers a different configuration. Despite the limited FOV, the relatively large lens aperture increases the light entry and leads to high-resolution images. The lens is deformable under the contraction of ciliary muscles. This changes the focusing power and can bring the objects of interest in focus $[19,20]$. The depth perception of different objects positioned at different distances is thus allowed [21, 22].

To achieve wide-angle imaging and position detection using a planar image sensor, we introduce a reconfigurable optofluidic device that incorporates the Xenos peckii compound eye design and the mammalian eye design into one system. A large elastomer membrane is arranged on top of a planar array of apertures and a planar array of small mammalian lenses. The top membrane serves as an array of deformable prisms to enlarge the FOV. A planar image sensor is used for imaging. The device provides an overall FOV as large as $130^{\circ}$ with the focusing power of the mammalian small lens varying from 21.4 diopter to 124.9 diopter. The position estimation of the objects in a wide distance range is also demonstrated. The small device can be integrated into portable and miniature systems to expect extensive optical applications.

\section{MATERIAL AND METHOD}

\section{Principle and Design}

The optofluidic device combines the architectural design of both the Xenos peckii compound eye and mammalian eye. A transparent big membrane atop can be deformed to a dome shape with different radii of curvature. An array $(2 \times 2)$ of small lenses is positioned underneath the big membrane whose focusing powers can be adjusted to focus the objects at different depths. The pinhole mode of the optofluidic device with the image sensor is shown in Figure 1a. The optical axis of the small lenses is arranged axialsymmetrically around the center axis of the image sensor that acts as a field stop. When the top membrane remains flat, the initial FOV of the device $\left(\mathrm{FOV}_{\mathrm{i}}\right)$ is expressed as:

$$
\mathrm{FOV}_{\mathrm{i}}=2 \delta=2 \arctan \left(\frac{\mathrm{l}+\mathrm{w}}{\mathrm{v}}\right)
$$

where $\delta$ is half of the $\mathrm{FOV}_{\mathrm{i}} ; l$ is half width of the image sensor; $w$ is the horizontal distance from the optical center of the small lens to the center axis of the device; and $v$ is the vertical distance from the small lens to the sensor. When the top membrane is deformed, the maximal achievable viewing angle $\alpha$ of the device is expressed as:

$$
\alpha=2 \arcsin \left(\mathrm{n} * \sin \left[\arcsin \left(\frac{\sin (\delta+\theta)}{\mathrm{n}}\right)+\theta\right]\right)
$$

where $\theta$ is the slope angle of the bottom surface of the top membrane; and $n$ is the refractive index of the fluid. The maximal viewing angle increases with the increasing $\theta$, i.e., the increasing deformation of the top membrane. By combining the initial FOV and the FOVs at different top membrane deformations, a large overall FOV can be obtained.

The optofluidic device consists of three glass windows, a cylindrical chamber, a top polydimethylsiloxane (PDMS) membrane with four cylindrical posts bulged atop and microfluidic channels underneath, an iris layer, a bottom microfluidic channel layer, and a bottom PDMS membrane with four small cavities that (a)

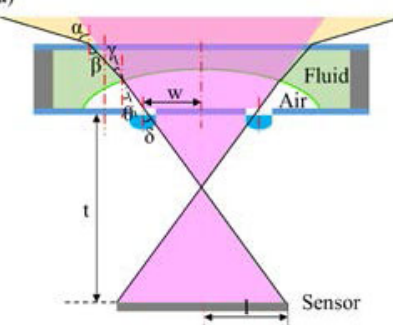

(b)

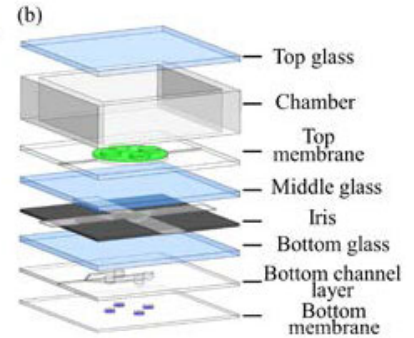

Figure 1: The configuration and optical schematic of the device. (a) The 2D optical layout. (b) The multi-layer schematic diagram. 
are arranged axial-symmetrically around the center of the cylindrical chamber (Figure 1b). The iris has two layers. The first layer consists of four films made of polymer dispersed liquid crystal (PDLC). The second layer consists of a black opaque film with four holes that have the same diameter. The locations of the four holes are the same as the four cavities on the bottom membrane. The four films are arranged axial-symmetrically around the center where each one covers the hole on the second opaque layer. When an AC voltage of $110 \mathrm{~V}$ is applied on the PDLC film, the second layer of the iris changes from opaque to transparent. Switchable imaging among the four individual small lenses can thus be implemented by applying selective electrical activation to the PDLC films. The bottom microfluidic channel and the bottom membrane forms the small lens array. The cylindrical chamber and the cavities of the small lens array are filled with an optical fluid whose refractive index (RI) matches that of PDMS.

\section{Fabrication and Assembly}

The cylindrical chamber was fabricated by replicating against a cylindrical acrylic post fabricated using laser engraving. The height of the cylindrical chamber was $7 \mathrm{~mm}$ and the diameter was $30 \mathrm{~mm}$. The top membrane, the bottom microfluidic channel layer and the bottom membrane were fabricated by replicating against the respective SU-8 molds that were pre-patterned with complementary features. The edge thickness of the top membrane was $100 \mu \mathrm{m}$ and the height of the four extruded posts on the top membrane was 600 $\mu \mathrm{m}$. The distance between the center of each post and the center of the top membrane was $4 \mathrm{~mm}$. The thickness of the bottom microfluidic channel layer was $400 \mu \mathrm{m}$. The thickness of the bottom membrane was $400 \mu \mathrm{m}$ and the diameter of the small lens on the membrane was $2 \mathrm{~mm}$. The distance between the center of each small lens and the center of the membrane is $3.5 \mathrm{~mm}$. All the layers were aligned and assembled under a microscope. The optical fluid was prepared by mixing glycerol with de-ionized water at a weight ratio of 3:2, and was instilled into the cylindrical chamber and the small lens cavities using two syringe pumps.

\section{RESULT AND DISCUSSION Optomechanical Simulation}

In this study, the top membrane serves as a prism to increase the FOV. As such, the bottom surface of the top membrane should be flat. However, the deformed surface of a thin PDMS membrane is often curvilinear, which may cause substantial optical aberration and deteriorate the image quality. To address this, four extruded posts were arranged on the top membrane. Optomechanical simulation was conducted to examine whether this design would help to reduce the curvature of the bottom surface. The top membranes with and without the extruded posts were deformed to the central deflections from $1 \mathrm{~mm}$ to $5 \mathrm{~mm}$. The flatness of the membrane was evaluated by calculating the slope angles at the lateral positions from $0.5 \mathrm{~mm}$ to $5.5 \mathrm{~mm}$ from the edge of the top membrane (Figure 2a). The results showed that under the same deflection the slope angle variance of the bottom surface with four extruded posts was significantly smaller than that without the posts. The bottom surface profiles were then imported into OpticStudio to obtain the RMS spot radius of the system at different incident angles (Figure 2b). The image plane was placed $11.5 \mathrm{~mm}$ away from the small lens. The result showed that when the center deflection of the top membrane reached $5 \mathrm{~mm}$, an overall FOV $>120^{\circ}$ can be obtained. The result also showed that the RMS spot radii of the system using the top membrane with the extruded posts were significantly smaller than that without the posts. This indicated that by, the system with the posts can yield better imaging performance.
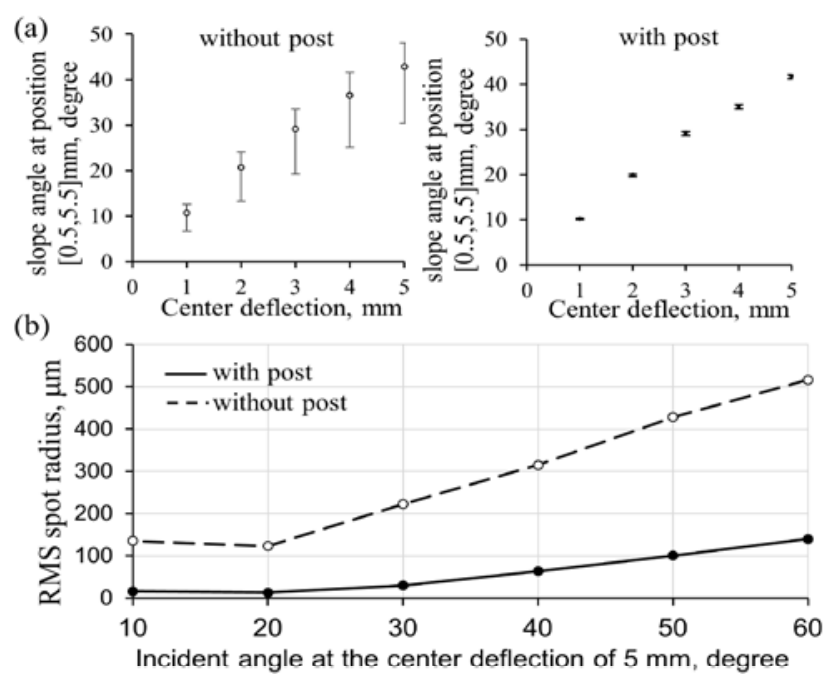

Figure 2: Optomechanical simulation. (a) The slope angle of the top membrane with and without the extruded posts. (b) The comparison of the RMS spot radii of the devices with and without the extruded posts. The center deflection of the top membrane was $5 \mathrm{~mm}$.

\section{Optical Power Measurement}

The optical power of the small lenses was varied by changing the differential pressure across the corresponding lens membrane. The change of the optical power upon the differential pressure was experimentally measured. The optical power changed from 21.4 diopter at the differential pressure of 80 mbar to 124.9 diopter at the differential pressure of 400 mbar (Figure 3). The resolution of the optical power is about 0.25 diopter given the minimal pressure increment of 1 mbar.

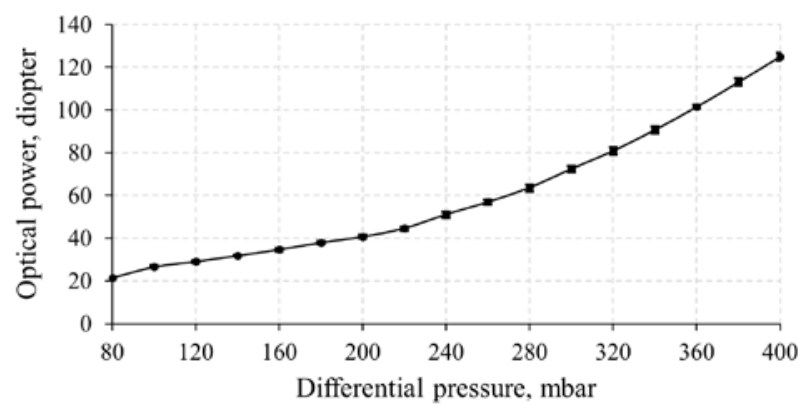

Figure 3: Tunable optical power of single small lens.

\section{FOV Measurement}

The FOV of the device was measured by viewing a target object that was placed $60 \mathrm{~mm}$ away from the device. A planar 1" CMOS image sensor (CMV4000-3E5, Point Grey) was used for image acquisition. The image sensor was placed $11.5 \mathrm{~mm}$ away on the other side of the device. The differential pressure across the top membrane was tuned from 0 to -450 mbar with the decrement of 50 mbar. The overall FOV changed from $82^{\circ}$ under the differential pressure of 0 mbar to the maximum of $130^{\circ}$ under the differential pressure of -450 mbar (Figure 4).

\section{Distortion Characterization}

The wide FOV can be obtained by the fisheye lenses. These lenses, however, have severe visual distortions, especially in the peripheral regions. The distorted images cannot be used for measurement purposes. Here, the distortions of the wide-angle images acquired by a fisheye lens and the reported wide-angle lens 
were compared. The distortion of the device and the fisheye lens was examined by imaging a dot array target. The array has 26 rows and 15 columns. The distance between the neighboring dots was 14 $\mathrm{mm}$. The target was placed $80 \mathrm{~mm}$ away from the device. The image distortion was represented by:

$$
\text { Distortion }(\%)=\left|\frac{\mathrm{AD}-\mathrm{PD}}{\mathrm{PD}}\right| \times 100
$$

where $\mathrm{AD}$ is the actual distance between the given dot to the center of the image, and PD is the predicted distance between the corresponding imaginary undistorted dot to the center of the image. The image of the dot array target by our device was formed by stitching individual images captured under the differential pressures across the top membrane of 0 mbar (corresponding to the FOV of $82^{\circ}$ ), -98 mbar (corresponding to the FOV of $100^{\circ}$ ), and $-296 \mathrm{mbar}$ (corresponding to the FOV of $120^{\circ}$ ), respectively (Figure 5a). The image by the fisheye lens was acquired using the same image sensor (Figure 5b). As shown, our device provides a significantly lower distortion than the fisheye lens, especially at the image peripheries. The maximal distortion of the images by the fisheye was $42 \%$, which was significantly higher than the maximal distortion of $16 \%$ by our device.

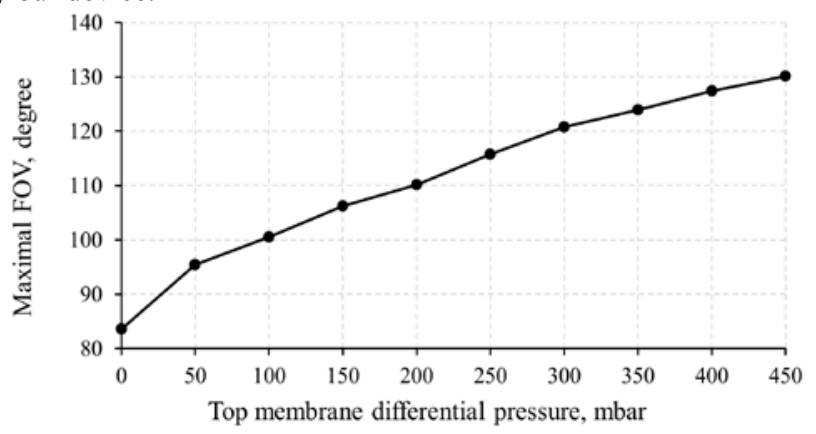

Figure 4: The Overall FOV of the device as a function of the differential pressure across the top membrane.
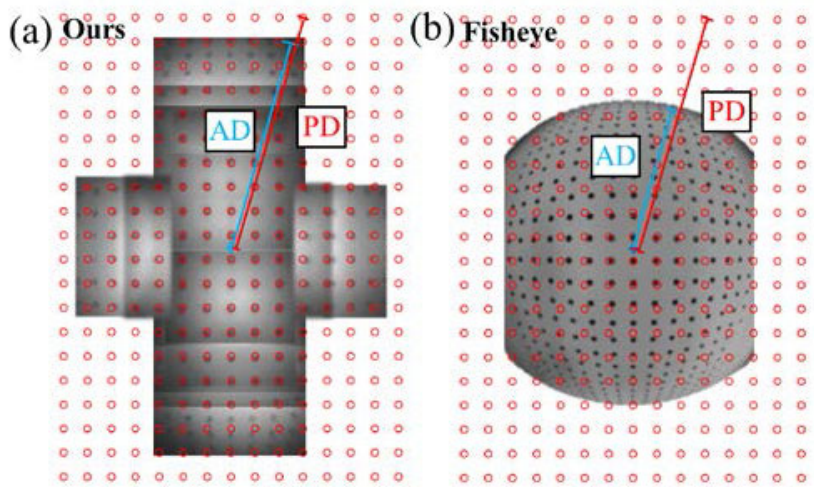

Figure 5: Distortion comparison of the reported device and a fisheye lens. Image of a dot array by (a) the device and (b) the fisheye lens.

\section{Wide-angle Imaging}

The wide-angle imaging capability was examined by imaging a picture of 'Last Supper' with a length of $340 \mathrm{~mm}$ and height of 65 $\mathrm{mm}$. The picture was placed $100 \mathrm{~mm}$ away from the device. The image sensor was placed $11.5 \mathrm{~mm}$ away on the other side of the device. The entire picture was covered within the horizontal FOV of $120^{\circ}$. During the imaging, the top membrane was deformed under the differential pressure across the top membrane of 0 mbar (corresponding to the FOV of $82^{\circ}$ ), -98 mbar (FOV of $100^{\circ}$ ), and 296 mbar (FOV of $120^{\circ}$ ), respectively. Under each FOV, the small lens was continuously tuned to get the different positions of the image in focus. On each image, only the image areas with a relatively high contrast were selected for image stitching. Such areas were adjusted to the same magnification and cropped to the same size. All the cropped neighboring images have 50\% overlapped regions. These images were stitched together to form a panorama of the picture (Figure 6a). The images taken with a fisheye lens and a single solid lens with the same aperture were also obtained for comparison (Figure 6b\&c). As seen, the image by the fisheye lens has significantly larger distortions at the peripheries of the image. The image by the solid lens had a significantly smaller FOV.

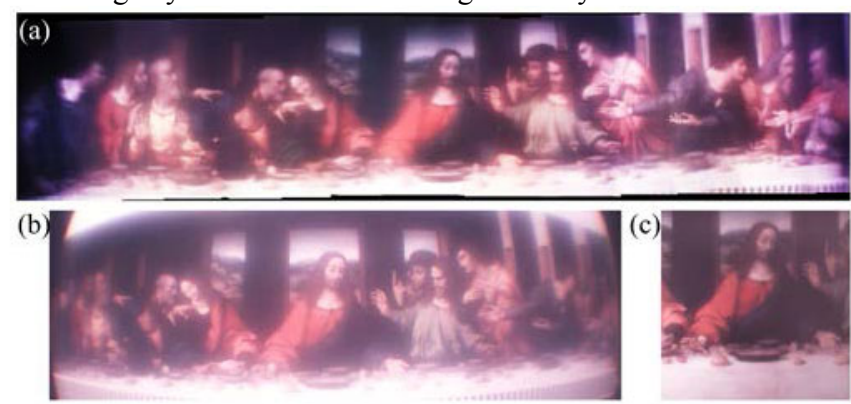

Figure 6: Wide-angle imaging comparison. Image of 'Last Supper' by (a) our device, (b) a fisheye lens, and (c) a solid lens. The three lenses had the same aperture.

\section{Position Estimation}

The capability of estimating the spatial positions of the objects was also demonstrated. According to the Snell Law calculation, the coordinates of an object were determined by four variables shown in Figure 7a, namely: the prism angle $\theta$, the displacement $d$, the optical power of the small lens, and the position of the object on the image sensor (point ' $A$ '). For analysis simplicity, the position of the object on the image sensor was fixed as about $1.88 \mathrm{~mm}$ from the edge of the image sensor. The optical power of the small lens was determined by the differential pressure across the small lens membrane. The prism angle $\theta$ and the displacement $d$ were both determined by the differential pressure across the top membrane.

Table 1: The Positions of the Nine Objects

\begin{tabular}{|c|c|c|}
\hline \multicolumn{3}{|c|}{$(\mathrm{X}, \mathrm{Y}), \mathrm{mm}$} \\
\hline$(17,120)$ & $(28,120)$ & $(39,120)$ \\
\hline$(13,90)$ & $(21,90)$ & $(29,90)$ \\
\hline$(9,60)$ & $(14,60)$ & $(19,60)$ \\
\hline
\end{tabular}

Nine objects were placed in the viewing space in front of the wide-angle lens, with the positions listed in Table 1. After bringing the images of the objects to the designed position ' $A$ ', the coordinates of the objects can be determined from the differential pressures across the top membrane and across the small lens membrane. Given the fact that the small lens had a depth of field and the measurement error in the prism angle membrane was not negligible, the point ' $A$ ' on the image plane corresponded to a small area in the viewing space, as plotted in Figure $\mathbf{7 b}$. The areas corresponding to the nine objects were estimated. As shown, all the nine objects fell into the areas estimated from the differential pressures. This indicated that the wide-angle lens can provide the accurate estimation of the object positions.

\section{CONCLUSION}

In this paper, we present a reconfigurable optofluidic lens that 
combines the architectural design of the Xenos peckii compound eye and the mammalian eye. A tunable FOV as large as $130^{\circ}$ was implemented using a planar image sensor. The image distortion of the lens was significantly lower than those of the fisheye lenses. Wide-angle imaging and position estimation were successfully demonstrated.

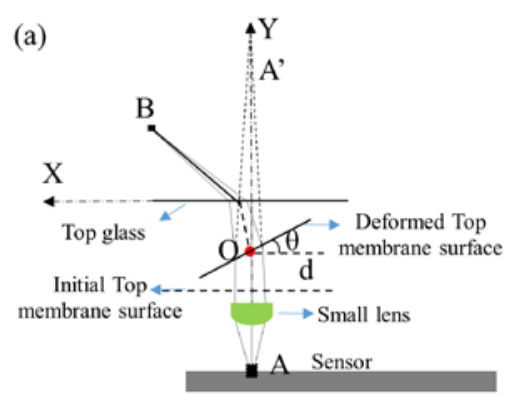

(b)

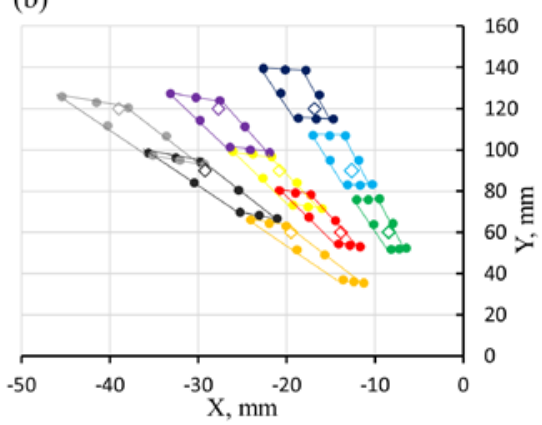

Figure 7: Position estimation. (a) The optical layout for position calculation; and (b) The position estimation of nine objects placed in the viewing space at different positions. The circles denote the actual position of the objects and the enclosed boxes indicate the estimated position ranges of the objects.

\section{ACKNOWLEDGEMENT}

The authors thank the support from National Science Foundation under the grant number 1509727. The authors also thank the graduate fellowship support from Pelotonia program.

\section{REFERENCES}

[1] A. Borst, "Drosophila's View on Insect Vision," Current Biology, vol. 19, pp. R36-R47, Jan 132009.

[2] A. Borst and J. Plett, "OPTICAL DEVICES Seeing the world through an insect's eyes," Nature, vol. 497, pp. 4748, May 22013.

[3] K. H. Jeong, J. Kim, and L. P. Lee, "Biologically inspired artificial compound eyes," Science, vol. 312, pp. 557-561, Apr 282006.

[4] R. Leitel, A. Brückner, W. Buß, S. Viollet, R. PericetCamara, H. Mallot, et al., "Curved artificial compoundeyes for autonomous navigation," in Micro-Optics 2014, 2014, p. $91300 \mathrm{H}$.

[5] Y. M. Song, Y. Xie, V. Malyarchuk, J. Xiao, I. Jung, K.J. Choi, et al., "Digital cameras with designs inspired by the arthropod eye," Nature, vol. 497, p. 95, 2013.

[6] R. Szema, J. Rastegar, and L. Lee, "An artificial compound eye for stereoendoscopy," Journal of medical engineering \& technology, vol. 28, pp. 117-124, 2004.

[7] J. Duparre, F. Wippermann, P. Dannberg, and A. Brauer, "Artificial compound eye zoom camera," Bioinspiration \& Biomimetics, vol. 3, Dec 2008.
[8] D. Floreano, R. Pericet-Camara, S. Viollet, F. Ruffier, A Brückner, R. Leitel, et al., "Miniature curved artificial compound eyes," Proceedings of the National Academy of Sciences, vol. 110, pp. 9267-9272, 2013.

[9] D. Keum, H. Jung, and K. H. Jeong, "Planar emulation of natural compound eyes," Small, vol. 8, pp. 2169-2173, 2012

[10] P. Qu, F. Chen, H. Liu, Q. Yang, J. Lu, J. Si, et al., "A simple route to fabricate artificial compound eye structures," Optics express, vol. 20, pp. 5775-5782, 2012.

[11] I. Jung, J. Xiao, V. Malyarchuk, C. Lu, M. Li, Z. Liu, et al., "Dynamically tunable hemispherical electronic eye camera system with adjustable zoom capability," Proceedings of the National Academy of Sciences, vol 108, pp. 1788-1793, 2011

[12] H. C. Ko, M. P. Stoykovich, J. Song, V. Malyarchuk, W. M. Choi, C.-J. Yu, et al., "A hemispherical electronic eye camera based on compressible silicon optoelectronics," Nature, vol. 454, p. 748, 2008.

[13] E. Buschbeck, B. Ehmer, and R. Hoy, "Chunk versus point sampling: visual imaging in a small insect," Science, vol. 286, pp. 1178-1180, 1999.

[14] D. Keum, D. Jeon, M. Kim, and K. Jeong, "Artificial compound eye inspired by imaging principle of Xenos peckii," in Solid-State Sensors, Actuators and Microsystems (TRANSDUCERS), 2015 Transducers2015 18th International Conference on, 2015, pp. 403406.

[15] W. Pix, J. M. Zanker, and J. Zeil, "The optomotor response and spatial resolution of the visual system in male Xenos vesparum (Strepsiptera)," Journal of Experimental Biology, vol. 203, pp. 3397-3409, 2000.

[16] M. Ma, F. Guo, Z. Cao, and K. Wang, "Development of an artificial compound eye system for three-dimensional object detection," Applied optics, vol. 53, pp. 1166-1172, 2014

[17] H. Zhang, L. Li, D. L. McCray, S. Scheiding, N. J. Naples, A. Gebhardt, et al., "Development of a low cost high precision three-layer 3D artificial compound eye," Optics express, vol. 21, pp. 22232-22245, 2013.

[18] J. Howard and A. Snyder, "Transduction as a limitation on compound eye function and design," Proc. R. Soc. Lond. B, vol. 217, pp. 287-307, 1983.

[19] Y. X. Yu, T. J. Zhang, A. Meadway, X. L. Wang, and Y. H. Zhang, "High-speed adaptive optics for imaging of the living human eye," Optics Express, vol. 23, pp. 23035 23052, Sep 72015

[20] K. Wei, H. Huang, Q. Wang, and Y. Zhao, "Focus-tunable liquid lens with an aspherical membrane for improved central and peripheral resolutions at high diopters," Optics Express, vol. 24, pp. 3929-3939, 2016.

[21] K. Wei, H. Zeng, and Y. Zhao, "Insect-Human Hybrid Eye (IHHE): an adaptive optofluidic lens combining the structural characteristics of insect and human eyes," Lab on a Chip, vol. 14, pp. 3594-3602, 2014.

[22] K. Wei and Y. Zhao, "A Three-Dimensional Deformable Liquid Lens Array for Directional and Wide Angle Laparoscopic Imaging," 26th IEEE International Conference on Micro Electro Mechanical Systems (MEMS 2013), pp. 133-136, 2013.

\section{CONTACT}

*Y. Zhao, tel: +1-614-247-7424; zhao.178@,osu.edu 